\title{
SYMMETRY FOR THE ENVELOPING ALGEBRA OF A RESTRICTED LIE ALGEBRA
}

JOHN R. SCHUE

In a recent paper [1], Berkson has shown that the restricted enveloping algebra $U$ of a restricted finite-dimensional Lie algebra $L$ is a Frobenius algebra. By requiring that each transformation in the adjoint representation of $L$ have zero trace (a condition satisfied by any nilpotent $L$ or any $L$ for which $[L, L]=L$ ) it turns out that $U$ is actually symmetric. A proof of this is given below.

We let $L$ be a restricted Lie algebra which is finite-dimensional over a field $K$ of characteristic $p>0$. For $x \in L$ let $D_{x}$ be defined on $L$ by $D_{x} y=[x, y]$, and let $\operatorname{Tr}\left(D_{x}\right)$ denote the trace of $D_{x}$. U will denote the restricted enveloping algebra of $L$ as defined and discussed in [2, pp. 185-192], and $U^{*}$ denotes the dual space of $U$ over $K$. For $u \in U$ and $\phi \in U^{*}$ define $u \phi$ and $\phi u$ by $(u \phi)(v)=\phi(v u),(\phi u)(v)=\phi(u v)$ for all $v \in U$. We choose a fixed ordered basis $x_{1}, \cdots, x_{n}$ of $L$ and thus $\left\{x_{1}^{i_{1}} \cdots x_{n}^{i_{n}}: 0 \leqq i_{j} \leqq p-1\right\}$ is a basis of $U$. For each such basis element of $U$ we define the degree as $\sum i_{j}$ and for a linear combination of basis elements define the degree as the maximum of the degrees of basis elements which appear with nonzero coefficients. Let $\phi_{0}$ be defined as the linear functional on $U$ which vanishes at each basis element except that $\phi_{0}\left(x_{1}^{p-1} \cdots x_{n}^{p-1}\right)=1$. The main result of [1] is that the linear mapping $u \rightarrow u \phi_{0}$ from $U$ to $U^{*}$ is one-one and onto. The result to be proved here is the following:

Theorem. $u \phi_{0}=\phi_{0} u$ for all $u \in U$ iff $\operatorname{Tr}\left(D_{x}\right)=0$ for all $x \in L$. Thus, if the latter condition is satisfied, $U$ is symmetric, i.e., the bilinear form $(u, v)=\phi_{0}(u v)$ is symmetric, nondegenerate, and $(u v, w)=(u, v w)$ for all $u, v, w$ in $U$.

The proof of the theorem will follow from several lemmas.

Lemma 1. Suppose $m \leqq n(p-1)$ and $y_{1}, \cdots, y_{m} \in L$. Then $\phi_{0}\left(y_{1}, \cdots, y_{m}\right)=\phi_{0}\left(y_{i_{1}}, \cdots, y_{i_{m}}\right)$ for any permutation $i_{1}, \cdots, i_{m}$ of $1, \cdots, m$. If $m<n(p-1)$ then $\phi_{0}\left(y_{1}, \cdots, y_{m}\right)=0$.

Proof. By using techniques like those used in [2] it follows that the degree of $y_{1}, \cdots, y_{m}$ is no greater than $m$ and that $y_{1}, \cdots, y_{m}$ $=y_{i_{1}} \cdots y_{i_{m}}+v$ where $v$ has degree less than $m$.

Received by the editors September 8, 1964 . 
LemMa 2. For $u$, v in $U$ let $[u, v]=u v-v u$. Then for $0 \leqq m<p$ and $x, y$ in $L,\left[x, y^{m}\right]=\sum_{1 m}^{m} C_{k}(-1)^{k_{y} m-k} D_{y}^{k}(x)$.

Proof. The proof is by induction on $m$. The case $m=1$ is immediate; we assume the result as stated to prove it for $m+1$. Now $\left[x, y^{m+1}\right]=\left[x, y^{m} y\right]=y^{m}[x, y]+\left[x, y^{m}\right] y=-y^{m} D_{y} x+y\left[x, y^{m}\right]$ $-\left[y,\left[x, y^{m}\right]\right]$. If the induction hypothesis is used on each of the last two terms, together with $\left[y, y^{m-k} D^{k} x\right]=y^{m-k} D_{y}^{k+1} x$, a straightforward computation will give the desired conclusion.

LEMMA 3. Let $u_{0}=x_{1}^{p-1} \cdots x_{n}^{p-1}$. For $x \in L$ we have $\phi_{0}\left(u_{0} x\right)=\phi_{0}\left(x u_{0}\right)$ $+\operatorname{Tr}\left(D_{x}\right)$.

PRoOF. Let $D_{x} x_{i}=\sum \lambda_{j i} x_{j}$. By virtue of Lemma $2, x_{i}^{p-1} x=x x_{i}^{p-1}$ $+x_{i}^{p-2}\left[x, x_{i}\right]+u_{i}$ where $u_{i}$ has degree less than $p-1$. From Lemma 1 we obtain $\phi_{0}\left(x_{1}^{p-1} \cdots x_{i}^{p-1} x \cdots x_{n}^{p-1}\right)=\phi_{0}\left(x_{1}^{p-1} \cdots x_{i}^{p-1} \cdots x_{n}^{p-1}\right)$ $+\sum \lambda_{j i} \phi_{0}\left(x_{1}^{p-1} \cdots x_{i}^{p-2} x_{j} \cdots x_{n}^{p-1}\right.$. However, since $x_{j}^{p} \in L$, each of the terms in the last summation is zero for $j \neq i$. Thus the sum reduces to $\lambda_{i 2} \phi_{0}\left(u_{0}\right)=\lambda_{i i}$. An induction argument can then be used to conclude that $\phi_{0}\left(u_{0} x\right)=\phi_{0}\left(x u_{0}\right)+\sum \lambda_{i i}=\phi_{0}\left(x u_{0}\right)+\operatorname{Tr}\left(D_{x}\right)$.

Proof of THE THEOREM. For each $u \in U$ there is a unique $u^{*} \in U$ such that $u^{*} \phi_{0}=\phi_{0} u$. The mapping $u \rightarrow u^{*}$ is clearly linear and is oneone for if $u^{*}$ is zero then $\left(v \phi_{0}\right)(u)=0$ for all $v \in U$ and this implies $u=0$. Moreover, it is an automorphism of the associative algebra $U$ since $(u v)^{*} \phi_{0}(w)=\phi_{0}(u v w)=\phi_{0}\left(v w u^{*}\right)=\phi_{0}\left(w u^{*} v^{*}\right)=\left(u^{*} v^{*}\right) \phi_{0}(w)$ for all $w$ implies $(u v)^{*}=u^{*} v^{*}$.

Suppose $\operatorname{Tr}\left(D_{x}\right)=0$ for all $x \in L$. Then $\phi_{0}\left(x u_{0}\right)=\phi_{0}\left(u_{0} x\right)$. From Lemma 1 we have $\phi_{0}(x u)=\phi_{0}(u x)$ for any basis element $u$ of smaller degree than $n(p-1)$. Hence the same equation holds for all $u$ and this implies $x^{*}=x$ for all $x \in L$. Since $U$ is generated by 1 and $L$ we have $u^{*}=u$ for all $u \in U$.

Conversely, if $u \phi_{0}=\phi_{0} u$ for all $u$ then $x=x^{*}$ for all $x \in L$ and Lemma 3 shows that $\operatorname{Tr}\left(D_{x}\right)=0$.

\section{REFERENCES}

1. Astrid J. Berkson, The u-algebra of a restricted Lie algebra is Frobenius, Proc. Amer. Math. Soc. 15 (1964), 14-15.

2. Nathan Jacobson, Lie algebras, Interscience, New York, 1962.

Macalester College 